\title{
Enabling Internet Singularity within the Electronic Commerce Trust Model
}

\author{
OMER MAHMOOD \\ School of Information Technologies, University of Sydney \\ and \\ SELVADURAI SELVAKENNEDY \\ School of Information Technologies, University of Sydney
}

Decentralization of publication is one of the great advantages of the Web's infrastructure. However, until now, Web failed to fully engage the user to contribute and add contents. This is the primary motivation for Web 2.0, which revolves around the notion that people can add contents for Collective Intelligence. Enterprises can also use Web 2.0 to reduce costs and increase profits through Social Network Analysis. Web 2.0 applications target to interconnect the users and contents so that the users can use contents to find participants and vice versa. Therefore, if the users are further assisted, to add contents to the web, link people with people, and link people with contents; then the distinction between the physical and electronic worlds will cease to exist. This web environment will enable the users to use web for Collective Intelligence and Social Network Analysis, defined here as 'Internet Singularity'. This paper proposes an application architecture based on decentralized information structure that links contents and people together. The architecture embraces FOAF, Atom, RDF, RDFS and OTER (Online Trust Evaluation RDF). OTER is constructed by using RDF Schema for linking and representing online trust evaluation information within Web 2.0. This architecture can be used as a model to develop Web 2.0 applications for any e-domain. However, it is believed that trust will be the main synthetic force in Web 2.0 as it is in the current physical environment. The proposed application architecture targets to assist the participants in committing online transactions while contributing towards Collective Intelligence.

Categories and Subject Descriptors: I.7.4 [Document and Text Processing]: Electronic Publishing; H.3.5 [Information Storage and Retrieval]: Online Information Services - Web-based services; Date sharing; K.4.4 [Computers and Society]: Electronic Commerce - Electronic data interchange (EDI); J.4 [Computer Applications]: Social and Behavioral Sciences - Sociology

General Terms: Human Factors, Design, Languages

Additional Key Words and Phrases: Semantic Web, Electronic Social Networks, Electronic Trust Evaluation, Human Factors in Computing

\section{INTRODUCTION}

Web 2.0 is not just about the birth of a set of new technologies per se. It is rather more related to how the technologies can be used to link the physical world with the electronic one within the social network domain to empower and facilitate users to contribute more. Web 2.0 is not only a set of technologies: it also has properties which aim for social integration, user-contributed content, user-generated metadata, transparent business processes and decentralized and participatory products and processes [Gartner, 2006a]

Within Web 2.0, the existence of social networks and the associated social network analysis (SNA) are considered to be of high significance. The resultant Collective and engineer web applications, so that the enterprises can increase revenues or save costs. Hinchcliffe [Hinchcliffe, 2006] proposed that Web 2.0 (in future) will lead to Internet

\footnotetext{
Authors' addresses: Omer Mahmood, Selvadurai Selvakennedy, School of Information Technologies, University of Sydney, NSW 2006, Australia; omer_mah@yahoo.com, s.kennedy@usyd.edu.au. Permission to make digital/hard copy of part of this work for personal or classroom use is granted without fee provided that the copies are not made or distributed for profit or commercial advantage, the copyright notice, the title of the publication, and its date of appear, and notice is given that copying is by permission of the ACM, Inc. To copy otherwise, to republish, to post on servers, or to redistribute to lists, requires prior specific permission and/or a fee.

(C) 2008 ACM 1529-3785/0700-0002 $\$ 5.00$
} 
Intelligence has been rated as transformational [Gartner, 2006b]. Collective intelligence is an intelligence that emerges from the collaboration and competition of many individuals with no centralized governing body. SNA involves the application of new ways to deploy Singularity. Flake [Flake, 2006] alluded that a deeper and tighter coupling between the online and offline worlds will expedite the growth of science, business and society, while quickening self-actualization. The author further predicted that with the passage of time, the Internet's content, composition and participants would more closely reflect and represent the physical world.

Based on the above findings, it may be acceptable to postulate that Internet Singularity is oneness of both the online and offline worlds. It is a reflection of the physical world, primarily facilitated by social computing. Such a social computing platform is likely to contain components that support and represent online social paradigms such as online identity, online reputation and online trust. To achieve Internet Singularity, web applications need to be modeled and developed, so that users are assisted to contribute contents and metadata. These contributions can be further used for collective intelligence and as SNA attributes.

In future web systems, trust will be the main synthetic force, as it is in the present physical environment. Trust based online merchants, independent rating systems, trusted peer to peer networks and personal electronic social networks will play a major role in reshaping the way business is conducted in the e-environment. Therefore, this paper concentrates on achieving Collective Intelligence in the domain of online initial trust evaluation in e-commerce through Internet Singularity.

This paper proposes a conceptual Web 2.0 application architecture that adopts Friend of a Friend (FOAF) [Brickley and Miller, 2005], Atom [Nottingham and Sayre, 2005], RDF and RDF Schema (RDFS) tools. A unique blend of these tools, as proposed here, will facilitate the online users to make decisions, while committing electronic transactions on the basis of initial trust evaluations learned from others. For this purpose, the model proposed in [Mahmood, 2006] is selected, as it outlines a suitable structure of metadata and provides a useful mathematical expression to evaluate the contents.

\section{TRUST AND ELECTRONIC COMMERCE}

Recently trust has been recognized as one of the main factors affecting electronic commerce. According to WISTA International E-Commerce Survey [WISTA, 2000], trust (26\%) is the most important barrier to electronic commerce in 27 surveyed countries. The survey recognized "trust as significant stumbling block in electronic commerce development due to the fact that electronic commerce is global and its international reach means that participants must deal with unknown or anonymous individuals and companies". The WISTA survey also identified payment security (25\%), trust in infrastructure (17\%) and information privacy (15\%) as the most important trust related issues for acceptance of electronic commerce. The survey established the impact of trust on electronic commerce with the majority of the respondents agreeing to this at least at a moderate level (strongly $42 \%$, moderately $35 \%$ ).

\subsection{Social Aspects Influencing Trust}

A connection needs to be established between electronic social networks and e-commerce through online initial trust. For this purpose, two physical social components are identified, and also found to be applicable in the electronic environment. These social 
components affecting user decisions in committing online transactions are discussed below.

2.1.1 Trust and Online Reputation. In the absence of trusted referral or past experience, online reputation can be one of the crucial factors for the user to establish relations with online service providers. Zacharia [Zacharia, 1999] states "reputation is usually defined as the amount of trust inspired by a particular person in a specific setting or domain of interest". Online reputation regarding an e-business is built by collating the past experiences of the users who have previously interacted with the same service provider. This technique, in the form of reviews, feedback and point ratings, is used by several online auction sites like eBay.com and some web retailers like Amazon.com to enhance users' level of trust on web merchants. However, under such circumstances, the users' level of trust in the information source plays a decisive role.

In an empirical study by Sarah et al. [2002], it was identified that most users would give high value to the previous customer endorsements, even more than third party affiliation, to judge the ability of the web merchant. In the same study, $80 \%$ of the respondents reacted positively to establishing trust on an online merchant, due to positive feedback from previous customers.

2.1.2 Trust and Trusted Referrals. Information regarding a product, physical or online business acquired from either the users' physical or online trusted social network, impacts the users' initial and subsequent level of trust in an online business. The impact is directly associated with the users' level of trust in the information in terms of the source's credibility, honesty and ability. Trusted referrals [Kim and Prabhakar, 2000] "are the primary means of disseminating market information when the services are particularly complex and difficult to evaluate. This implies that if one gets positive wordof-mouth referrals on e-commerce from a person with strong personal ties, the consumer may establish higher levels of initial trust in e-commerce". According to Fullam et al. [2004], the users' belief on accuracy and certainty conveyed in the information ultimately determines their level of trust in the information source.

Accordingly, the two recognized social electronic components that link together the electronic social network with the electronic commerce are illustrated in figure 1.

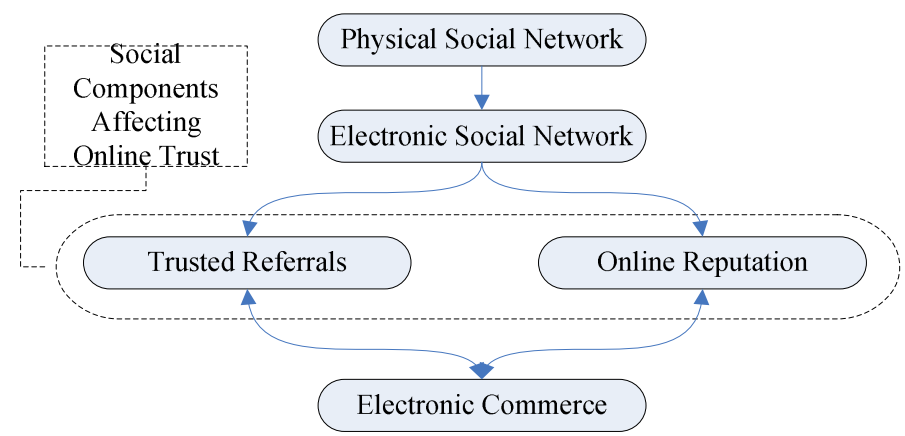

Figure 1: Recognised links between physical social network and e-commerce.

\section{INITIAL TRUST EVALUATION IN ELECTRONIC COMMERCE}

Mahmood [Mahmood, 2006] proposed a mathematical model to evaluate trust in the electronic environment, which comprises trust in the electronic transaction and the e- 
business entity. Trust in online business is further subdivided into subjective probabilities consisting of trust in the business entity's performance and honesty. User perceived trust in a transaction impacts and controls the exchange of funds. The following figure, adopted from the selected trust evaluation model, outlines the involved dynamics of trust in an electronic transaction:

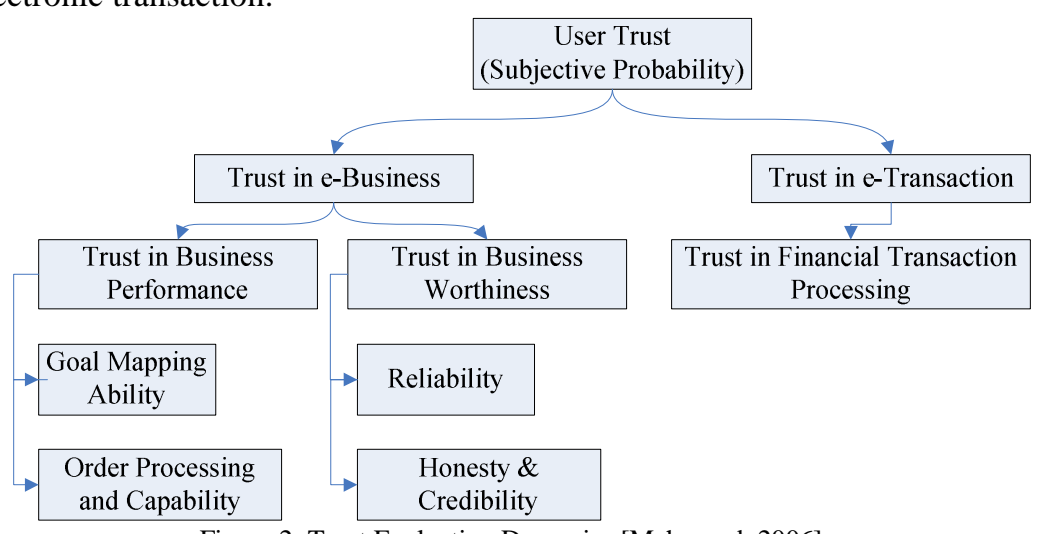

Figure 2: Trust Evaluation Dynamics [Mahmood, 2006].

The same author proposed the use of the following mathematical expression to determine the worthiness of an electronic transaction for an online service:

Where

$$
W o I=\left(\left(\frac{w_{b p}+w_{b h}}{w_{p}+w_{h}}\right)-r w_{u i}\right) * 100
$$

WoI = worthy of investment

$w_{b p}=$ weighted trust in business performance

$w_{b h}=$ weighted trust in business honesty

$r w_{u i}=$ the ratio of weighted probability of losing uninsured investment

$w_{p}=$ Subjective weight of business performance

$w_{h}=$ Subjective weight of business honesty

All the above used weighted values are computed as follows:

Weighted trust in business performance $\left(w_{b p}\right)=p_{b} * w_{p}$

Weighted trust in business honesty $\left(w_{b h}\right)=p_{h} * w_{h}$

Where

While $r w_{u i}$ is computed as $\frac{\left(1-p_{t}\right) * f_{u i} * w_{t}}{m_{w t}}$

$p_{t}=$ subjective probability of financial transaction processing

$w_{t}=$ subjective weight of financial transaction processing 


$$
\begin{aligned}
& m_{w t}=\text { represents the maximum weight which can be assigned to financial } \\
& \text { transaction processing. ' } m_{w t} \text {, is fixed at } 10 \\
& f_{u i}=\text { fraction of uninsured investment. Which is computed as } \frac{t_{i}-i_{i}}{t_{i}} \text {. Where } \\
& t_{i}=\text { total investment and } i_{i} \text { is insured investment amount }
\end{aligned}
$$

The computed WoI value can be used by the online user to obtain an indication of whether they should complete an online transaction or not, on the basis of the user assigned weights and subjective probabilities. To compute WoI for each online transaction, the use of a browser plugin was proposed [Mahmood, 2006] so that the user can enter necessary data in order.

\section{CONCEPTUAL LINKING OF INDIVIDUAL TRUST FOR COLLECTIVE INTELLIGENCE}

Since the above trust evaluation model lacks in metadata and content sharing, it is not compatible with the Internet Singularity vision. The web application architecture, proposed in this paper, enables online users to store and share the subjective probabilities, weights and transaction values so that the contributed information can be used for collective intelligence. The proposed application architecture is divided into three main modules, as described in the following subsections.

\subsection{Information Sharing Module}

Atom Syndication Format 1.0 has been selected for information sharing, which is an XML language used for web feeds. Two obvious choices for this role were RSS and Atom. However, Atom 1.0 is used on the basis of its advantages over RSS as outlined in table 1 .

Table I: Comparison of Atom 1.0 and RSS 


\begin{tabular}{|l|l|}
\hline \multicolumn{1}{|c|}{ Atom 1.0 } & \multicolumn{1}{|c|}{ RSS } \\
\hline $\begin{array}{l}\text { Atom is defined within XML Namespace } \\
\text { [Atom Namespace, 2005]. }\end{array}$ & $\begin{array}{l}\text { RSS is not defined within XML } \\
\text { Namespace. }\end{array}$ \\
\hline $\begin{array}{l}\text { Atom has standardized autodiscovery. } \\
\text { Atom has registered IANA MIME type } \\
\text { 'application/atom+xml'. }\end{array}$ & $\begin{array}{l}\text { RSS uses many non standard variants of } \\
\text { autodiscovery. RSS 2.0 feeds are often } \\
\text { sent as application/rss+xml', although it is } \\
\text { not a registered MIME-type. }\end{array}$ \\
\hline $\begin{array}{l}\text { Atom uses the Atom Publishing Protocol } \\
\text { (APP). APP is a simple HTTP-based } \\
\text { protocol for creating and updating Web } \\
\text { resources. APP is a IEFT draft protocol. }\end{array}$ & $\begin{array}{l}\text { MetaWeblog and Blogger are the two } \\
\text { popular APIs widely used with RSS. } \\
\text { However, they are not interoperable. }\end{array}$ \\
\hline $\begin{array}{l}\text { Atom syndication format is published as } \\
\text { an IETF standard in RFC 4287 [IEFT, } \\
\text { 2005]. }\end{array}$ & $\begin{array}{l}\text { RSS has multiple incompatible and widely- } \\
\text { adopted variants. }\end{array}$ \\
\hline
\end{tabular}

\subsection{Social Representation Module}

Friend of a Friend (FOAF), a Resource Description Framework (RDF) Vocabulary, has been utilized to store, share and represent social arrangements of a user. The FOAF vocabulary is identified by the namespace URI ' $h t t p: / / x m l n s . c o m / f o a f / 0.1 /$ ' and enables individuals and organizations to participate in creating an open network of their trusted friends, including both individuals and organizations. The FOAF project looks into ways to use machine readable and parseable web pages for people, groups, companies and web applications. To protect the user and its friends from phishing attacks, the proposed application saves the emails in SHA1 encoded format by using 'foaf:mbox_sha1sum' element.

\subsection{Trust Evaluation Module}

Since RDF is one of the languages of Web 2.0, RDF and RDF Schema (RDFS) have been identified and used for each user's trust ratings and storage. A new RDF schema termed Online Trust Evaluation - RDFS (OTE-RDFS), has been engineered for this purpose, which uses the Dublin Core defined elements, IANA and W3C Standards and ISO standards. The standards include the use of ISO8601:2004 [ISO8601, 2004] for date format, ISO639-2 [Library of Congress, 2006] for language definition, DCMI Vocabulary [DCMI, 2006] for genre of the resource, ISO4217:2001 [BSI, 2005] for currency code specification and IANA media types [IANA, 2006] for service output. The conceptual diagram of suggested OTE-RDFS is depicted in figure 3. To avoid clutter, most of the underlying technical information has been omitted from the figure. 


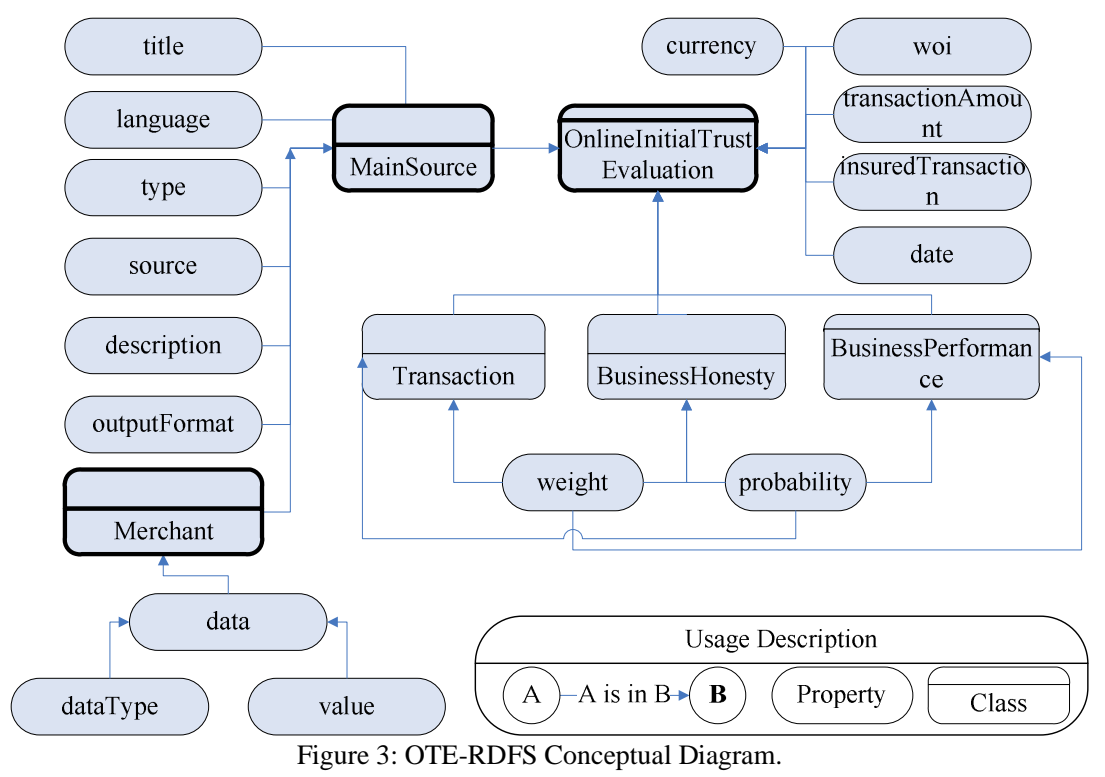

\section{CONNECTING FOAF AND OTER THROUGH ATOM}

As discussed earlier, to feed the contents of trust evaluations (OTER) and user's FOAF social network information, Atom 1.0 has been used. The proposed Atom document structure is described further.

\subsection{Feed Information}

The feed information section provides a description of the Atom document and the author's related information. The 'updated' element is used to specify the last modification date and time of the Atom document.

\subsection{FOAF Information}

Inside the 'feed' element, each user's category of FOAF is represented by using the 'entry' element. Within entry, the 'category' element is used to specify the type of the entry. It has a 'term' attribute, which specifies the type of document such as "FOAF" and a 'scheme' attribute, which points to namespace such as 'http://xmlns.com/foaf/0.1' for FOAF documents. Moreover, a link to FOAF document is also embedded inside the 'entry' element, and 'application/rdf $+\mathrm{xml}$ ' is assigned to the 'type' attribute for the ease of processing. Besides above, the 'published' element is used to specify the publication/creation date and time of the FOAF document.

\subsection{OTER Information}

The user's online trust evaluation document is also linked with the Atom document by using the 'entry' element. Within entry, the type of the entry is specified by 'category' element. For example "OTER" is specified as the value of the 'term' attribute, and the 'scheme' attribute points to OTE-RDFS document. Moreover, an OTER document also embeds a link inside the 'entry' element and sets a 'type' attribute to 


\section{8 - O. Mahmood and S. Selvakennedy}

'application/rdf $+\mathrm{xml}$ ' to assist auto-processing. The last modification date is specified by the 'updated' element, and the publication/creation date and time of the corresponding OTER document is specified by the 'published' element.

Figure 4 outlines the connections between FOAF, OTER, OTE-RDFS documents and the user through the user's Atom document.

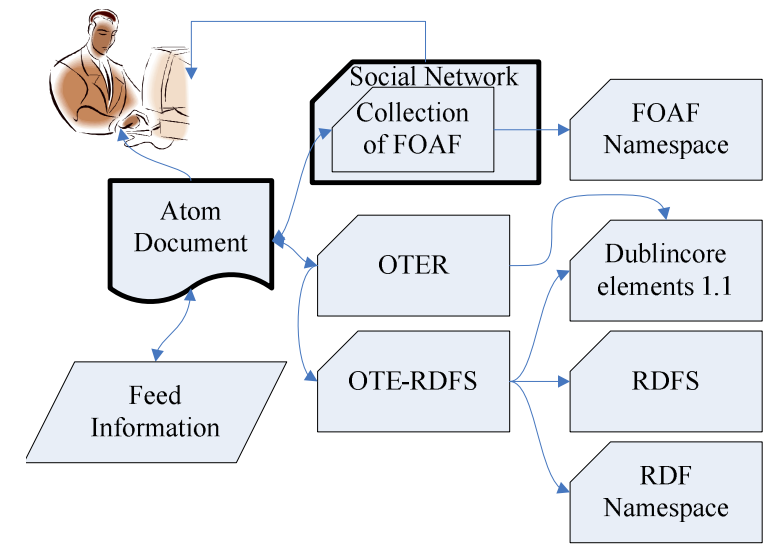

Figure 4: Linking the components of the proposed system through Atom

\section{SOCIAL NETWORKING WITH FOAF}

The proposed architecture divides the FOAF document into Person and Group sections. The Person section of the FOAF document provides brief information about the author and links to the author's Atom and OTER documents. In the Group section, after specifying the group name, the 'member' element and the 'Person' class of FOAF namespace are used to specify information regarding each member of the group. Within each 'Person' class, links to the OTER and Atom documents of each member are specified by the 'Document' class. This ensures backward linking of the member's FOAF document with FOAF and Atom documents.

Figure 5 presents an example scenario where a user's FOAF document connects two people. The FOAF link also enables the users to access other users' Atom and OTER documents, where each OTER document provides trust evaluation information on multiple e-merchants. Besides these links, each Atom document is also structured to refer to OTER and multiple FOAF documents, so that the search can be performed recursively. 


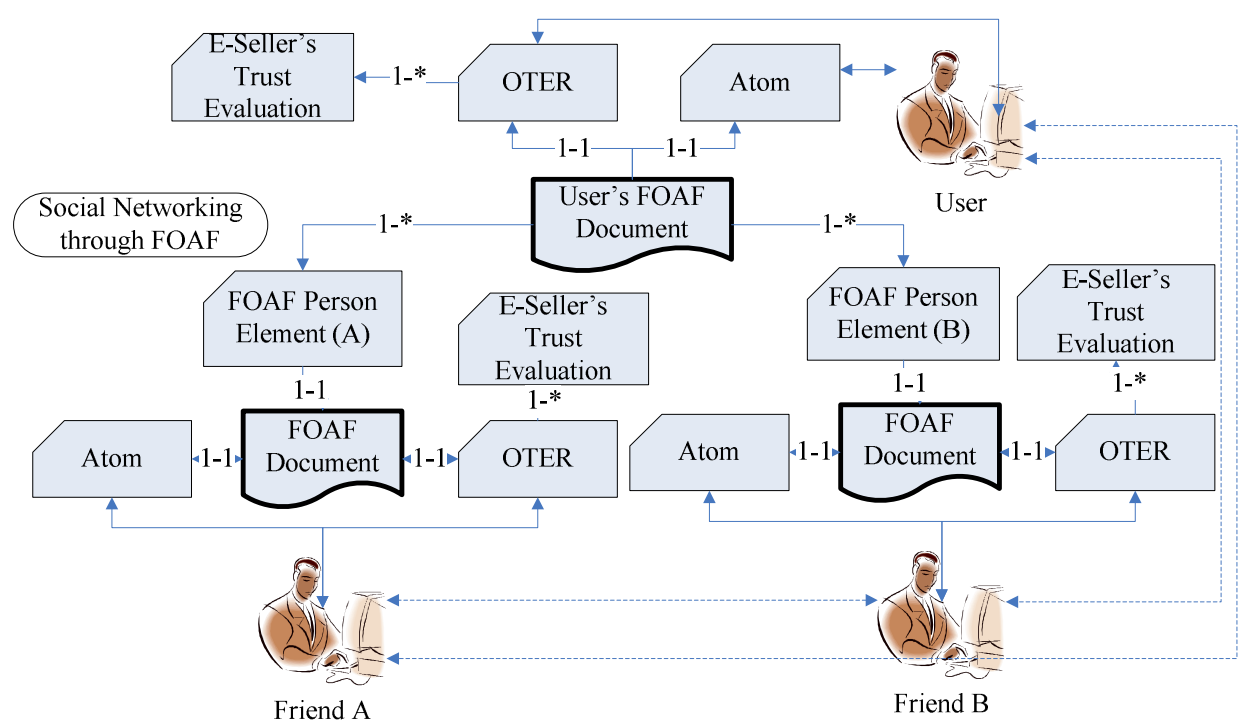

Figure 5: Linking users and OTER Documents through FOAF.

Direct connections between the FOAF, Atom and OTER documents are established in the architecture. Therefore, such an architecture enables the participants to use contents to find people and use people to find contents (see figure 6), as desired by the Internet Singularity vision.

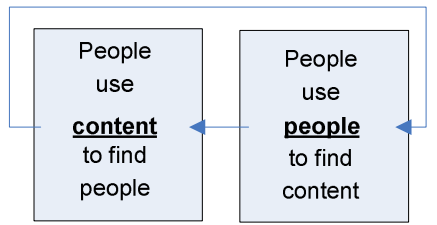

Figure 6: Interconnecting People with Contents to assist collaboration.

\section{RESTRICTING YOUR PERSONAL DATA}

Web is an open place and generally users do not want to share their personal (private) information with public. For example, a user may want to share his name, home page information and picture publicly, but wishes to restrict access to phone contact details to certain parties. For such situations, the user can use PGP utilities like OpenPGP [GPG4Win, 2006] to encrypt and sign private contents of both OTER or FOAF documents by using their public key. Such contents can only be decrypted by using the user's private key. The user can then publish the encrypted and signed RDF GPG (rdf.gpg) documents publicly by linking them with OTER, Atom or FOAF documents with the help of the RDFS property 'seeAlso' and the Web of Trust namespace [XmlNs, 2002]. The user can now distribute his private key to the desired parties so that they can decrypt the private encrypted signed documents.

\section{CONCEPTUAL MODEL OF INFORMATION FLOW}

Figure 7 presents the conceptual diagram that outlines the sequential flow of information between the application and the user, as this paper only concentrates on the information 
flow. It does not cover the user interface and mechanism of information gathering, since these have been already addressed in detail in [Mahmood, 2006], and is outside the scope of this paper.

When the plugin is active, it checks for current merchant's trust evaluation information from the user's OTER document. If the information is available in the user's OTER file (step 5), then it is displayed to the user (step 6a). The user can also change and update his record (steps 9a). However, if there is no data on current merchant in the user's OTER document (step 6b), then the user is prompted to make evaluations and enter the subjective trust evaluation values (step 7b). If the user feels confident in making evaluations and enters the trust data, then the user's OTER file is updated (step 9bi). However, if the user feels that he/she needs assistance from the FOAF social network (step 9bii), then a query is submitted to the FOAF network (step 10b). The search is performed in a recursive manner [1]. Initially, the OTER files of the user's FOAF social network are searched. This is followed by the search of the OTER files of each corresponding person's FOAF network and so forth. At this point, the application enables the user to limit the search by specifying the depth of current search. Once the search is performed, the trust evaluation data is gathered and processed (step 11). All of the trust values are represented to the user as a mean of their set (step 12). The user then evaluates the computed mean values and decides to either accept them or change them before adding a new record in his/her personal OTER document (step 14). Since the proposed information architecture uses Atom as the main linking document, each addition of record or alteration of data in the user's OTER generates a notification to all the users or services which have subscribed to the user's Atom feed (step 10a).

\footnotetext{
${ }^{1}$ Complete source code and all the resources related to this project can be accessed from http://oter.sourceforge.net
} 


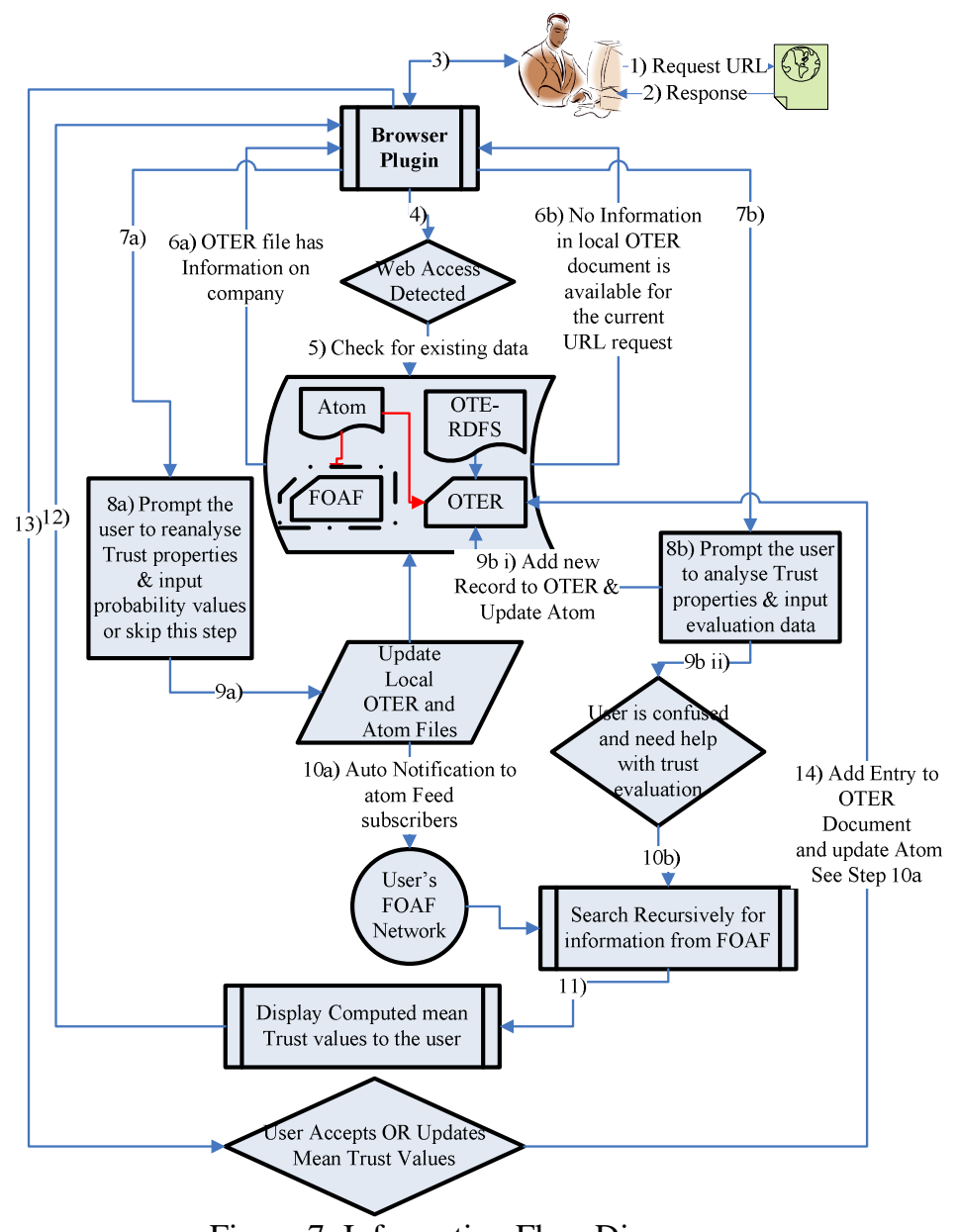

Figure 7: Information Flow Diagram.

\section{FEASIBILITY OF THE ARCHITECTURE}

The architecture is based on a decentralized storage and access model. There is no need for a central server for user registration, aggregation and processing of data. Any use of a centralized server would be against the philosophy of Web 2.0 and such an application would also lose the crucial advantage of the web's ability to support decentralized publications. Since the collection, linking and processing of information is completely distributed, the proposed architecture is more failsafe and robust.

\section{CONCLUSION AND FUTURE DIRECTIONS}

The application architecture proposed in this paper uses decentralized information storage and access structure, while keeping the contents and the people interlinked. The architecture uses Web 2.0 technologies such as Atom, FOAF, OTER and RDFS for data storage, representation, processing and sharing. An online trust evaluation RDF (OTER) scheme is designed, which is an application of the proposed online trust evaluation RDF Vocabulary (OTE-RDFS). The components and overall architecture fully conforms to Web 2.0 standards and aims to assist the participants in committing online transactions. The architecture establishes a strong link between the contents and people thus enabling 
users to utilize web for Collective Intelligence. This unique method of structuring and linking Atom 1.0, FOAF, RDF and RDFS can be used in any electronic domain to achieve 'Internet Singularity'. In addition, the application architecture can also be used as a blueprint for general Web 2.0 applications.

Currently, independent modules are being developed and used to generate, process and represent FOAF and trust evaluation information to fine tune the model. However, in the future, all modules can be merged to develop a browser plugin as the main access point to the application, and to enhance the users' contribution for collective intelligence.

\section{REFERENCES}

BRICKLEY, D. \& MILLER, L. 2005. FOAF Vocabulary Specification. W3C Retrieved from http://xmlns.com/FOAF/0.1/on 16 September 2006

BRITISH STANDARDS INSTITUTE (BSI). 2005. BSI Currency Code Service - ISO 4217 Maintenance Agency. BSI. Retrieved from http://www.bsi-global.com/British_Standards/currency/index.xalter on 23 September 2006

DCMI. 2006. DCMI Type Vocabulary Retrieved from http://dublincore.org/documents/dcmi-type-vocabulary/ on 23 September 2006

FLAKE, G.W. 2006. How I Learned to Stop Worrying and Love the Imminent Internet Singularity, Microsoft (24th January, 2006) Retrieved from http://research.microsoft.com/workshops/fs2006/presentations/19_Flake_071706.ppt on 18th September 2006

Fullam K., BARBER K.S. 2004. A Belief Revision Algorithm Based on information valuation. Laboratory for Intelligent Processes and Systems. Technical Report TR2003-UT-LIPS-021

GARTNER. 2006a. Gartner Says Web 2.0 Offers Many Opportunities for Growth, But Few Enterprises Will Immediately Adopt All Aspects Necessary for Significant Business Impact. Gartner.com, Retrieved from http://www.gartner.com/press_releases/asset_152253 11.html on 18th September 2006

GARTNER. 2006b. Gartner's 2006 Emerging Technologies Hype Cycle Highlights Key Technology Themes. Gartner.com, Retrieved from http://www.gartner.com/it/page.jsp?id=495475 on 18th September 2006

GPG4WIN. 2006. GPG for Windows Utility, including WinPT. Retrieved from http://www.gpg4win.org/ on 24 September 2006

HiNCHCLIFFE, D. 2006. Thinking Beyond Web 2.0: Social Computing and the Internet Singularity. Web 2.0 Web Services Journal,Retrieved from http://web2.wsj2.com/thinking_beyond_web_20_social_computing_and_the_internet_sin.htm on 19th September 2006

IANA. 2006. MIME Media Types. IANA Retrieved from http://www.iana.org/assignments/media-types/ on 23 September 2006

ISO. 2004. ISO 8601 - 2004 Retrieved from http://www.iso.org/iso/en/CatalogueDetailPage.CatalogueDetail?CSNUMBER=40874 on 23 September 2006

KIM, K. AND PRABhaKaR, B. 2000. Initial Trust, Perceived Risk, and the Adoption of Internet Banking. In Proceedings of the Twenty First International Conference on Information Systems, December 2000.537 543

Library of Congress. 2006. Codes for the Representation of Names of Languages. Library of Congress. Retrieved from http://www.loc.gov/standards/iso639-2/php/code_list.php on 2 December 2006

MAHMOOD, O. 2006. Modelling Trust Recognition and Evaluation in Electronic Environment. International Journal of Networking and Virtual Organisations. Special Issue on "Trust for Virtual Organisations and Virtual Teams". Inderscience Publishers Ltd. ISSN (Online): 1741-5225, ISSN (Print): 1470-9503 (In Press)

NotTingham, M \& SAYRE, R. 2005. The Atom Syndication Format. Internet Society Taskforce (IEFT) FRC 4287 Retrieved from http://tools.ietf.org/html/rfc4287 on 19 September 2006

SARAH P. W. S, CHOON-Ling S, KaI H. L. 2002. A Preliminary Assessment of Different Trust Formation Models: The Effect of Third Party Endorsements on Online Shopping, In Proceedings of the 36th Hawaii International Conference on System Sciences (HICSS'03)

THE INTERNET SOCIETy. 2005. The Atom Syndication Format, Internet Official Protocol Standards, RFC 4287 , December 2005. Retrieved from http://tools.ietf.org/html/rfc4287 on 19 September 2006

W3C. 2005. Atom Syndication Format, Namespace. W3C, December 2005, Retrieved from http://www.w3.org/2005/Atom on 21 September 2006 
WORLD INFORMATION TECHNOLOGY AND SERVICES ALLIANCE (WISTA). 2000. In World information technology and services alliance (WISTA) International survey of E-Commerce 2000 Retrieved from http://www.witsa.org/papers/EComSurv.pdfon 26 August 2006

XMLNs. 2002. Web of Trust (WOT) Namespace, xmlns.com. Retrieved from http://xmlns.com/wot/0.1/ on 24 September 2006

ZACHARIA, G. 1999. Trust management through reputation mechanisms. In Proceedings of the Second Workshop on Deception, Fraud and Trust in Agent Societies, Seattle. Retrieved August, 2006163 - 167. from http://www.istc.cnr.it/T3/download/aamas1999/Zacharia.pdf

Received November 2006; revised December 2007; accepted December 2007 